\title{
EFFECTIVENESS OF NON PHARMACOLOGICAL THERAPY ON BED WETTING IN CHILDREN
}

\author{
Veena Prasad $^{1 *}$, Nutan Singh ${ }^{2}$ \\ ${ }^{1}$ Deptt of Pediatrics, UFHT medical college, Haldwani, Distt- Nainital (Uttarakhand), India \\ ${ }^{2}$ Deptt of Pediatrics, UFHT medical college,Haldwani, Distt-Nainital (Uttarakhand), India
}

\begin{abstract}
:
Nocturnal enuresis (bedwetting) involuntary wetting during sleep without any inherent suggestion of frequency of bedwetting or pathophysiology. Bedwetting is a widespread and distressing condition that can have a deep impact on a child or young person's behaviour, emotional wellbeing and social life. There are a number of different disturbances of physiology that may be associated with bedwetting. These disturbances may be categorised as sleep arousal difficulties, polyuria and bladder dysfunction. A prospective observational study was carried out in children with the age group of 4 to 12 years with complaints of bed wetting visiting the OPD of UFHT medical college Haldwani Uttaranchal during 2008-2009. In our study of two years out of 114 cases $88(72.19 \%)$ were in the age group of 4 to 6 years. Children are generally expected to be dry at night by developmental age of 5 years, and historically it has been a common practice not to offer advice to families of children who are younger than 5 years and are bedwetting. The children less than 6 years responded well to the non pharmacological measures. Encouraging results were seen in the age group of 6 to 8 years with a cure rate of $78.57 \%$ and a cure rate of $85.71 \%$ and $100 \%$ in age group of 8 to10 and 10 to 12 years respectively.

Keywords: Children, enuresis, emotional, social life and physiology.
\end{abstract}

\section{INTRODUCTION}

Nocturnal enuresis (bedwetting) involuntary wetting during sleep without any inherent suggestion of frequency of bedwetting or pathophysiology. Bedwetting is a widespread and distressing condition that can have a deep impact on a child or young person's behaviour, emotional wellbeing and social life. It is also very stressfull for the parents or carers. 1 The causes of bedwetting are not fully understood. Bedwetting can be considered to be a symptom that may result from a combination of different predisposing factors. There are a number of different disturbances of physiology that may be associated with bedwetting. These disturbances may be categorised as sleep arousal difficulties, polyuria and bladder dysfunction. Bedwetting also often runs in families primary nocturnal enuresis. This is the recurrent involuntary passage of urine during sleep by a child aged 5 years or older, who has never achieved consistent night-time dryness. This may further be subdivided into children who have enuresis only at night and those who also have daytime symptoms (urgency, frequency, or daytime wetting). Secondary nocturnal enuresis . This is the involuntary passage of urine during sleep by a child who has previously been dry for at least 6 months ${ }^{2}$. The causes of bedwetting are not fully understood. Bedwetting can be considered to be a symptom that may result from a combination of different predisposing factors. There are a number of different disturbances of physiology that may be associated with bedwetting. These disturbances may be categorised as sleep arousal difficulties, polyuria and bladder dysfunction. Bedwetting also often runs in families ${ }^{3}$. Risk factorsThere are a number of factors that predispose to persistent nocturnal enuresis. There is a genetic pre disposition ${ }^{4}$ As an illustration, it is said that the risk of nocturnal enuresis is $15 \%$ if neither parent was affected, $40 \%$ if one parent was affected and $75 \%$ if both had the condition $^{5} .23 \%$ of nocturnal enuresis is associated with encopresis and daytime incontinence ${ }^{6}$. Enuresis is to be expected as a manifestation of developmental delay, in those syndromes. Even without gross developmental delay, there is more likely to be persistent bedwetting in children with delayed developmental milestones, premature delivery or behavioural disorders such as hyperactivity or inattention deficits ${ }^{7,8}$.

\section{MATERIAL AND METHODS}

A prospective observational study was carried out in children with age more than 4 to 12 years with complaints of bed wetting visiting the OPD of UFHT medical college Haldwani Uttaranchal during 20082009.

The following definitions were used for this guideline: ${ }^{9}$ - Bedwetting: involuntary wetting during sleep without any inherent suggestion of frequency of bedwetting or pathophysiology.

- Daytime symptoms: daytime urinary symptoms such as wetting, urinary frequency or urgency.

- Response to an intervention: the child has achieved 14 consecutive dry nights or a $90 \%$ improvement in the number of wet nights per week.

- Partial response: the child's symptoms have improved but 14 consecutive dry nights or a $90 \%$ improvement in the number of wet nights per week has not been achieved.

*Corresponding Author

Veena Prasad

Professor, Deptt of Pediatrics UFHT medical college, Haldwani Distt- Nainital (Uttarakhand) 
Urinalysis routinely in children and young people with bedwetting was done, in cases where-

1. Bedwetting started in the last few days or weeks.

2. There are daytime symptoms

3. There are any signs of ill health

4. There is a history, symptoms or signs suggestive of urinary tract infection

5. There is a history, symptoms or signs suggestive of diabetes mellitus.

Assessment of any co morbid conditions was done. e.g

1. Constipation and/or soiling

2. Developmental, attention or learning difficulties

3. Behavioural or emotional problems

4. Family problems or a vulnerable child or young person or family.

\section{Mode of treatment}

1. Dry bed training: Dry bed training consists of a strict schedule of waking the child at night, attempting to condition the child into waking by himself/herself10 Studies show this training is ineffective by itself $^{11}$ and does not increase the success rate when used in conjunction with a bedwetting alarm. ${ }^{12}$

2. Using an alarm

3. Lifting and waking

4. Advice on fluid intake, diet and toileting pattern. Advise children and young people with bedwetting and their parents or carers that: adequate daily fluid intake is important in the management of bedwetting daily fluid intake varies according to ambient temperature, dietary intake and physical activity. Advise the child or young person and parents or carers that the consumption of caffeine-based drinks should be avoided in children and young people with bedwetting. Advise the child or young person and parents or carers to eat a healthy diet

5. Psychological counselling of parents, caretakers and patients.

6. Reward systems

7. Star chart: A star chart allows a child and parents to track dry nights, as a record and/or as part of a reward program. This can be done either alone or with other treatments. There is no research to show effectiveness, either in reducing bedwetting or in helping self-esteem ${ }^{11}$. Some psychologists, however, recommend star charts as a way to celebrate successes and help a child's self-esteem ${ }^{12}$.

8. Punishment for bedwetting should be discouraged. Medical literature states and studies show that punishing or shaming a child for bedwetting will frequently make the situation worse. Doctors describe a downward cycle where a child punished for bedwetting feels shame and a loss of self-confidence. This can cause increased bedwetting incidents, leading to more punishment and shaming ${ }^{13}$

\section{RESULT:}

Two years study (2008-2009) was done in patients coming in OPD in UFHT Medical College Haldwani, Uttarakhand. 114 patients age more than 4 years were included in our study.

Table 1: Number Patients both sex in different age groups

\begin{tabular}{|l|l|l|l|l|}
\hline Age group(years) & Male & Female & Total & Percentage $(\%)$ \\
\hline $4-6$ & 56 & 32 & 88 & 77.19 \\
\hline $6-8$ & 08 & 06 & 14 & 12.28 \\
\hline $8-10$ & 04 & 03 & 07 & 06.14 \\
\hline $10-12$ & 03 & 02 & 05 & 04.39 \\
\hline Total & 71 & 43 & 114 & 100.00 \\
\hline
\end{tabular}

Table 2: Monthly follow up 4-6 years age groups patients

\begin{tabular}{|c|c|c|c|}
\hline \multicolumn{3}{|c|}{ Effectiveness of non pharmacological therapy } \\
\hline Monthly Follow up in age (4-6 yrs) & No response & Partial response & Complete response \\
\hline $1^{\text {st }}$ follow up & 63 & 25 & 00 \\
\hline $2^{\text {nd }}$ follow up & 45 & 35 & 08 \\
\hline $3^{\text {rd }}$ follow up & 11 & 40 & 37 \\
\hline
\end{tabular}

Complete response in the age group 4-6 years after 3 months was achieved in $42.04 \%$.

Table 3: Monthly follow up 6 - 8 years age groups patients

\begin{tabular}{|c|c|c|c|}
\hline \multicolumn{3}{|c|}{ Effectiveness of non pharmacological therapy } \\
\hline Monthly Follow up in age $(6-8$ yrs $)$ & No response & Partial response & Complete response \\
\hline $1^{\text {st }}$ follow up & 5 & 8 & 1 \\
\hline $2^{\text {nd }}$ follow up & 2 & 9 & 3 \\
\hline $3^{\text {rd }}$ follow up & 1 & 2 & 11 \\
\hline
\end{tabular}

Complete response in the age group 6-8 years after 3 months was achieved in $78.57 \%$.

Table 4: Monthly follow up 8-10 years age groups patients

\begin{tabular}{|c|c|c|c|}
\hline \multicolumn{3}{|c|}{ Effectiveness of non pharmacological therapy } \\
\hline Monthly Follow up in age $(8-10$ yrs $)$ & No response & Partial response & Complete response \\
\hline $1^{\text {st }}$ follow up & 1 & 4 & 2 \\
\hline $2^{\text {nd }}$ follow up & 0 & 3 & 4 \\
\hline $3^{\text {rd }}$ follow up & 0 & 1 & 6 \\
\hline
\end{tabular}

Complete response in the age group 8-10 years after 3 months was achieved in $85.71 \%$. 
Table 5: Monthly follow up 10 - 12 years age groups patients

\begin{tabular}{|c|c|c|c|}
\hline \multicolumn{3}{|c|}{ Effectiveness of non pharmacological therapy } \\
\hline Monthly Follow up in age (10-12yrs) & No response & Partial response & Complete response \\
\hline $1^{\text {st }}$ follow up & 0 & 2 & 3 \\
\hline $2^{\text {nd }}$ follow up & 0 & 1 & 4 \\
\hline $3^{\text {rd }}$ follow up & 0 & 0 & 5 \\
\hline
\end{tabular}

Complete response in the age group 10-12 years after 3 months was achieved in $100 \%$.

\section{DISCUSSION \& CONCLUSION}

In our study of two years out of 114 cases $88(72.19 \%)$ were in the age group of 4 to 6 years, $14(12.28 \%), 7$ $(6.14 \%)$ and $5(4.39 \%)$ cases in the age groups 6 to 8,8 to 10 , and 10 to 12 years respectively. Similar results have been seen in the study conducted by Wan et al, At five years of age, 15 to 25 percent of children wet the bed. With each year of maturity, the percentage of bedwetters declines by 15 percent. Hence, 8 percent of 12 year-old boys and 4 percent of 12-year-old girls are enuretic, only 1 to 3 percent of adolescents are still wetting their bed ${ }^{[14]}$.

Children are generally expected to be dry at night by a developmental age of 5 years, and historically it has been common practice not to offer advice to families of children who are younger than 5 years and are bedwetting. The guideline scope did not specify a minimum age limit to allow consideration of whether there are interventions of benefit to younger children previously excluded from advice and services due to their age. We have included specific advice for children under 5 years, and indicated treatment options for children between 5 and 7 years. ${ }^{[3]}$ In our study we found

\section{REFERENCES}

1. Butler RJ, Heron $\mathbf{J}$. The prevalence of infrequent bedwetting and nocturnal enuresis in childhood: A large British cohort. Scandinavian Journal of Urology and Nephrology 2008;42:257-64

2. Bedwetting (enuresis); NICE CKS, January 2010.

3. NICE guideline, The management of bedwetting in children and young people $\backslash$ Issued: October 2010 NICE clinical guideline 111 guidance.nice.org.uk/cg111

4. WangQW, Wen JG, ZhuQH, et al; The effect of familial aggregation on the children with primary nocturnal enuresis.Neurourol Urodyn. 2008 Nov14.

5. Backwin H. The genetics of enuresis. In: Colvin I, McKeith, RC, Meadow, SR, eds; Philadelphia: Lippincott, 1973.

6. Sureshkumar P, Jones M, Caldwell PH, et al; Risk Factors for Nocturnal Enuresis in School-Age Children. J Urol. 2009.

7. Touchette E, Petit D, Paquet J, et al; Bed-wetting and its association with developmental milestones in early childhood.Arch Pediatr Adolesc Med. 2005;159(12):1129-34.

8. Elia J, Takeda T, Deberardinis R, et al; Nocturnal enuresis: a suggestive endophenotype marker for a subgroup of J Pediatr. 2009;155(2):239-44. that the children less than 6 years responded well to the non pharmacological measures. Encouraging results were seen in the age group of 6 to 8 years with a cure rate of $78.57 \%$ and a cure rate of $85.71 \%$ and $100 \%$ in age group of 8 to10 and 10 to 12 years respectively. The most important aspect of treatment is determining if the child is motivated to become dry. This is especially true for behavioral management ${ }^{[16]}$. If it is determined that a child is not motivated to become dry, treatment should be postponed or simplified until the child is ready. ${ }^{[15]}$ failure rate encountered in our study was due to lack of self motivation of patients and parents, confidence in the approach and poor compliance of parents due to sleep disturbance caused by measures such as alarms, lifting and waking the child .

Most paediatricians do not consider bedwetting to be a problem until a child is at least six years of age ${ }^{[15]}$ but in our study non pharmacological measures in the age group 4 to 6 years has achieved a success rate of $42.04 \%$, hence early intervention should be recommended. The most important thing to remember is that with care and perseverance, nocturnal enuresis is a problem that can be successfully treated.

9. Nocturnal enuresis: The management of bedwetting in children and young people,NOCTURNAL ENURESIS: FINAL VERSION 2010; 9

10. American Family Physician. Retrieved 2008-02-03.

11. Evans, Jonathan. "Evidence based paediatrics: Evidence based management of nocturnal enuresis". BMJ. Retrieved 2008-0203 .

12. Fackler, Amy. "Dry-bed training for bed-wetting". Yahoo! Health. Retrieved 2008-02-03.

13. "Bedwetting". Retrieved 2009-09-12.

14. Wan J, Greenfield S. Enuresis and common voiding abnormalities. Pediatr Clin North Am. 1997; 44:1117-31.

15. Bennett HJ. Waking Up Dry: A Guide to Help Children Overcome Bedwetting. Elk Grove Village, IL: American Academy of Pediatrics, 2005.

16. Hjalmas K, Arnold T, Bower W, et al. Nocturnal enuresis: An international evidence based management strategy. Journal of Urology 2004; 171:2545-2561. 